\title{
Laboreal
}

Volume $13 \mathrm{~N}^{\circ} 1$ | 2017

Varia

\section{Hablemos de nuestro trabajo. Riesgos psicosociales en entornos de trabajo de extensionistas agropecuarios de la Argentina}

Vamos falar sobre o nosso trabalho. Fatores psicossociais de risco em contextos de trabalho dos técnicos do setor agropecuário da Argentina

Parlons de notre travail. Facteurs de risque psychosociaux dans des contextes de travail de techniciens de l'agriculture en Argentine

Let's talk about our work. Psychosocial risk factors in the working contexts of agricultural technicians in Argentina

\section{Rossana Cacivio}

\section{OpenEdition}

Journals

Edición electrónica

URL: http://journals.openedition.org/laboreal/1886

DOI: 10.4000/laboreal. 1886

ISSN: 1646-5237

Editor

Universidade do Porto

Referencia electrónica

Rossana Cacivio, «Hablemos de nuestro trabajo. Riesgos psicosociales en entornos de trabajo de extensionistas agropecuarios de la Argentina », Laboreal [En línea], Volume $13 N^{0} 1$ | 2017, Publicado el 01 julio 2017, consultado el 06 octubre 2019. URL : http://journals.openedition.org/laboreal/1886 ; DOI : 10.4000/laboreal.1886

Este documento fue generado automáticamente el 6 octubre 2019.

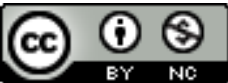

Laboreal está licenciado com uma Licença Creative Commons - Atribuição-NãoComercial 4.0 Internacional. 


\section{Hablemos de nuestro trabajo. Riesgos psicosociales en entornos de trabajo de extensionistas agropecuarios de la Argentina}

Vamos falar sobre o nosso trabalho. Fatores psicossociais de risco em contextos de trabalho dos técnicos do setor agropecuário da Argentina

Parlons de notre travail. Facteurs de risque psychosociaux dans des contextes de travail de techniciens de l'agriculture en Argentine

Let's talk about our work. Psychosocial risk factors in the working contexts of agricultural technicians in Argentina

\section{Rossana Cacivio}

\section{NOTA DEL EDITOR}

http://dx.doi.org/10.15667/laborealxiii0117rc

Manuscrito recibido en: Enero/2017

Aceptado tras peritaje: Junio/2017

\section{Introducción}

1 La Agronomía, y dentro de ella la extensión agropecuaria es una profesión que se caracteriza por la gran complejidad de los problemas que resuelve dada la naturaleza de la agricultura, dependiente de aspectos sociales, económicos, tecnológicos, culturales, políticos y ambientales. El extensionista debe identificar, diagnosticar e intervenir en un contexto de incertidumbre, debiendo desarrollar las competencias 
necesarias para actuar, que - dada la multidimensionalidad de las situaciones que resuelve- muchas veces exceden su formación.

2 En los últimos 30 años los profesionales que acompañamos desde la formación el trabajo de los servicios de extensión -en pos del Desarrollo Rural- hemos asistido a sucesivas transformaciones de la acción pública en los territorios rurales en función de los cambios políticos que se sucedieron en la Argentina. En ese contexto hemos formado e intercambiado experiencias con distintas camadas de extensionistas, relevando y dando respuesta a sus necesidades formativas, según la época.

este periodo, la tendencia de los sistemas de extensión públicos consistió en asistir mayoritariamente a productores familiares, desde la incipiente empresa familiar capitalizada hasta aquellos en situación de subsistencia o autoconsumo, dejando en manos de las organizaciones y técnicos del sector privado el trabajo con audiencias más empresariales.

4 Los sucesivos intercambios entre productores y extensionistas forjaron un vínculo estrecho entre ambos que se tradujo en confianza y un profundo conocimiento de las formas de decidir del productor, tanto en cuestiones productivas como de socialización. Esta confianza acercó al extensionista a la lógica del productor y lo convirtió en un traductor de las inquietudes, necesidades e insatisfacciones del hombre de campo.

5 Con el tiempo, el paulatino estrechamiento de los márgenes de producción, requirió revisar las estrategias de toma de decisiones e incorporar nuevas habilidades de gestión ya que las demandas excedían los conocimientos de ambos actores. Así los extensionistas tuvieron que desarrollar nuevas competencias de organización y gestión de empresas, asociaciones entre ellas, financiamientos y pronósticos de mercados que excedían su formación de grado.

6 En muchos casos el productor no visualizó los cambios económicos que se sucedían, produciéndose una crisis a nivel familiar y productivo, quedando ambos sistemas más expuestos a las contingencias económicas. El extensionista acompañó estas evoluciones quedando íntimamente ligado a las decisiones que se tomaron en dichos contextos, siendo testigo de los aciertos y fracasos de muchas familias y establecimientos agropecuarios.

\section{Pregunta de Investigación}

El seguimiento de estos procesos nos permitió observar durante los primeros años que los profesionales carecían, en general, de un enfoque sistémico que les permitiera comprender los procesos de forma interdependiente y dinámica, a la vez que disponían de una baja instrumentación en tecnologías sociales con las cuales abordar la complejidad de las políticas de integración vertical y horizontal propuestas desde los programas estatales de intervención para productores agropecuarios. La pregunta que nos hicimos fue: ¿Qué les pasa que se los nota tan estresados?

8 Existe la tendencia en las organizaciones laborales de ver al stress, la ansiedad y la explicitación de las emociones en general, como "un conocimiento incómodo" y consecuentemente se ha propendido a evitarlo o ignorarlo. En "Trabajar para ser feliz" (Baudelot \& Gollac, 2011) se habla de cierta ideología viril que atraviesa determinadas profesiones, entre ellas la agricultura, el trabajo rural y sus profesiones, donde estos actores reaccionan al stress con más trabajo, endureciendo sus posiciones y apelando a 
la "hombría" para controlar las emociones que les genera el trabajo. Vemos también a muchas mujeres que ejercen la extensión en organizaciones del sector agropecuario Argentino quedar atrapadas en los paradigmas generados por estas culturas corporativamente masculinas.

9 Esta "demanda implícita" sobre el stress de los profesionales, nos llevó a abrir espacios en diferentes organizaciones donde poner en situación propuestas de capacitación que aportaran a la construcción de un perfil más polivalente del extensionista, complementando la formación en tecnologías productivas, con aquellos conocimientos e instrumentos que les permitieran dar respuesta a las nuevas demandas de los sistemas productivos agroindustriales.

10 Trabajamos estas dimensiones en cursos optativos de grado académico, proyectos de extensión universitaria, cursos de posgrado, Maestrías, trabajos de Consultoría y fundamentalmente a través de capacitaciones profesionales en agencias estatales y privadas.

11 En esos espacios y durante el proceso nos sorprendió que muchos de los emergentes grupales hablaran de la falta de reconocimiento del esfuerzo realizado durante su labor, muchas veces exigidos por mostrar resultados y productos concretos, sin ser reconocidos por el enorme proceso que desarrollan en el territorio.

12 La creciente complejidad de las demandas expuso a estos profesionales a elevados niveles de stress y frustración. Entendimos que para reconocer la dimensión colectiva del problema, los extensionistas debían utilizar los espacios de capacitación, intercambiar sus experiencias, hacer converger las perspectivas individuales, reconocer los universales que atravesaban cada relato y así revisar sus prácticas profesionales en forma conjunta. De este modo pudieron explicitar la tensión en sus trabajos, ver procesos más generales que su experiencia particular y fundamentalmente darle una voz a cada caso.

\section{Materiales y Métodos}

13 Una de las cuestiones que más nos ocupó durante la investigación fue poder abordar desde distintas dimensiones la complejidad del tema en cuestión, por eso decidimos utilizar una metodología de tipo cualitativa que, a través de una batería de instrumentos, pudiera relevar y sistematizar la situación de cada uno de los profesionales que participaron del proceso e integrar sus particularidades en una conclusión conjunta.

14 Así, propusimos una investigación descriptiva del modo como perciben las condiciones de trabajo los extensionistas agropecuarios que pretende darnos una visión general, aproximativa sobre este recorte de la realidad, ya que el estudio de los factores psicosociales de los riesgos profesionales registra escasos antecedentes en nuestro país, menos aún explorado y reconocido en extensionistas agropecuarios, sobre el que es difícil formular hipótesis precisas o de cierta generalidad dada la diversidad de los actores.

15 Al ser un diseño principalmente emergente, durante el recorrido estuvimos abiertos a lo inesperado, a revisar y modificar imágenes mentales y conceptos según la marcha del proceso. También nos volvimos más flexibles a través del dialogo e interacción con los 
participantes, ya que durante el proceso de indagación el diseño fue sufriendo cambios que, suponemos, enriquecieron y volvieron más original el resultado final.

Esta plasticidad le dio al recorrido una forma espiralada, abriéndose tanto al diseño de la propuesta escrita, como al proceso recorrido hasta llegar a las conclusiones finales.

Priorizamos escuchar y retratar el punto de vista de los extensionistas, lo cual significó asumir un abordaje metodológico "más comprensivo que explicativo" (Honoré, 2002), promoviendo una mayor atención a sus enunciados y apelando a una concepción de salud en el trabajo más abarcativa y multidimensional, en la que interactúan las dimensiones física, psicológica y social, dándole más visibilidad a los "pequeños" problemas de salud y reforzando la noción de "bienestar en el trabajo" (Maggi, 2006).

De hecho, la integración de cuestiones relativas al contexto de trabajo, que reflejan una dimensión más subjetiva de la actividad (Gollac \& Volkoff, 2000) constituyeron una de las preocupaciones centrales del estudio y un punto de anclaje permanente en el abordaje metodológico propuesto.

19 A través del registro de las diversas miradas que aportaron los participantes generamos información detallada - que respalda las conclusiones - y esperamos que aporte una mayor comprensión al tema estudiado y pueda ser objeto de un trabajo analítico posterior.

20 Triangulamos diversos métodos y técnicas, complementando resultados tanto cualitativos como cuantitativos, que si bien fueron construidos a partir de abordajes metodológicos distintos, no son mutuamente excluyentes. Así aumentamos la confianza en los datos; esperando compensar algún sesgo que pueda impregnar nuestra subjetividad.

21 Durante el proceso de investigación cualitativa se ha logrado un ambiente de confianza con los entrevistados, a quienes se les aseguró la confidencialidad de sus datos y opiniones, garantizando en consecuencia la seguridad de los procedimientos a través de la utilización de estándares de trabajo, pautas de escritura y registro de los datos textuales, diferenciando entre testimonios e interpretaciones del investigador.

Debido a la forma cooperativa en que hemos construido el conocimiento con los encuestados y entrevistados afirmamos la confirmabilidad de los datos y dejamos constancia de su validación comunicativa, ya que los entrevistados han hecho una lectura crítica de las transcripciones de las entrevistas y visado sus encuestas, evaluando la calidad de las descripciones, el relevamiento de todas las perspectivas y la captación de su significado.

Por último, como nuestro propósito es contribuir a la práctica de la extensión y de alguna manera calibrar una actividad desgastante, esperamos haber contribuido al mejoramiento de las condiciones de trabajo de los extensionistas a través del aporte de un modelo teórico que pueda ser transferible a otros extensionistas y perfiles profesionales similares.

\subsection{Estrategias de recogida de información}

24 Como estrategia de recogida de información hemos utilizado: encuestas, entrevistas, observaciones, así como la confección de documentos y grabaciones. Utilizamos tanto datos cualitativos como cuantitativos, predominando los primeros debido a su 
adecuación para captar los significados simbólicos que emergen en la interacción social.

Los cuatro (4) instrumentos/técnicas utilizados fueron la Encuesta, el Análisis de correspondencias múltiples, las Entrevistas semiestructuradas y la Clínica de la actividad.

\subsubsection{Encuesta}

A la hora de elegir el instrumento para realizar la encuesta y medir los factores de riesgo, hicimos una investigación profunda de la oferta disponible evaluando sus beneficios y restricciones.

En general, los nuevos cuestionarios utilizados para evaluar los riesgos psicosociales del trabajo ya integran las dimensiones personal y social de la actividad del trabajo, ahora reforzadas por la incorporación de otros factores psicosociales, como las nuevas formas de relación salarial y de horarios, la conciliación entre la vida profesional y la vida privada, entre otros que, aparentemente más apartados de la caracterización de la actividad en el trabajo permiten comprender, también, la dimensión emocional que le está asociada (Davezies, 2009).

Si bien sabíamos que los diseños de estos cuestionarios sintetizan preguntas que ya han sido analizadas y discutidas en estudios de campo previos, no es obvio que sean condición suficiente para garantizar que los análisis estadísticos emergentes revelen los vínculos entre salud y trabajo si no se facilita la expresión y explicación de lo vivido por el trabajador.

De hecho, la integración de un conjunto diverso de cuestiones relativas al contexto de trabajo, como aquellas que reflejan una dimensión más subjetiva de la actividad, constituyen una de las preocupaciones centrales en estos cuestionarios y un punto de anclaje permanente en el abordaje metodológico propuesto (Gollac \& Volkoff, 2000).

\section{Cuestionario CoPSoQ-ISTAS21}

Utilizamos el Método CoPSoQ-ISTAS 21, Cuestionario de Evaluación de Riesgos Psicosociales en el Trabajo que es la adaptación realizada por el Instituto Sindical de Trabajo, Ambiente y Salud de España (ISTAS, 2003) del Cuestionario Psicosocial de Copenhague (CoPsoQ), instrumento que fue diseñado para la investigación, evaluación y prevención de los riesgos psicosociales, desarrollado por el National Research Centre for the Working Environment (NRCWE, 2000).

31 El CoPsoQ-ISTAS 21, es un cuestionario de aplicación individual, que evalúa al trabajador frente a la exposición de factores de riesgo para la salud de naturaleza psicosocial mediante las respuestas anónimas, de todo el colectivo empleado en la unidad objeto de evaluación. Es un instrumento de evaluación orientado a la prevención, cuyo objetivo es proporcionar valores en relación a una muestra de referencia, y plantear soluciones que puedan aplicarse en las organizaciones. (Moncada, Llorens, \& Kristensen, 2002; Moncada, Llorens, Navarro, \& Kristensen, 2005).

Existen tres versiones de este instrumento: una larga, diseñada para grandes investigaciones; una media, para la evaluación de riesgos en medianas (25 o más trabajadores) y grandes empresas; y otra corta, para trabajar en pequeños grupos. Si bien la versión larga es la ideal para investigación, su desarrollo dura más de dos horas 
y sólo es aplicable con aquellos empleados en relación de dependencia, dentro del marco laboral. La versión corta es la que utilizamos para los cursos de posgrado como las cohortes de la Maestría Procesos Locales de Innovación y Desarrollo Rural (PLIDER) y los otros grupos encuestados ya que puede realizarse en una hora y trabajar posteriormente sobre una reflexión de los resultados obtenidos. factores de riesgos para la salud de naturaleza psicosocial a los que está expuesto el trabajador en cada situación concreta de trabajo, denominados "Apartados"; los rangos de puntuación para cada Apartado están diseñados estadísticamente y permiten tanto introducir una dimensión cuantitativa al estudio como traducirlo fácilmente a colores en el sentido de un semáforo y tener una visión inmediata del nivel de riesgo psicosocial donde verde es sin riesgo, amarillo alerta y rojo alto riesgo. 
Cuadro 1: Cuestionario CoPSoQ-ISTAS 21. Colores y rangos de puntuaciones por Apartado

\begin{tabular}{|c|c|c|c|}
\hline nage & 100CAA000 & 0000259990 & 599B1B0.em \\
\hline \multirow{2}{*}{$\begin{array}{l}\text { Apartado/Dimensión } \\
\text { psicosocial }\end{array}$} & \multicolumn{3}{|c|}{$\begin{array}{l}\text { Puntuaciones para la población ocupada de } \\
\text { referencia }\end{array}$} \\
\hline & Verde & Amarillo & Rojo \\
\hline 1. Exigencias psicológicas & 0 a 7 & 8 a 10 & 11 a 24 \\
\hline $\begin{array}{l}\text { 2. Trabajo activo y } \\
\text { posibilidades de desarrollo }\end{array}$ & 40 a 26 & 25 a 21 & 20 a 0 \\
\hline 3. Inseguridad & 0 a 1 & 2 a 5 & 6 a 16 \\
\hline $\begin{array}{l}\text { 4. Apoyo social y calidad de } \\
\text { liderazgo }\end{array}$ & 40 a 29 & 28 a 24 & 23 a 0 \\
\hline 5. Doble presencia & 0 a 3 & 4 a 6 & 7 a 16 \\
\hline 6. Estima & 16 a 13 & 12 a 11 & 10 a 0 \\
\hline
\end{tabular}

A lo largo de cinco (5) años se realizó la encuesta a un total de152 profesionales (92 hombres y 60 mujeres) extensionistas agropecuarios de distintas organizaciones y lugares del país. Los mismos estaban aglomerados en nueve (9) grupos, cada uno encabezado por el nombre de la localidad donde habían sido encuestados (Patagones, La Plata, Balcarce, Rosario, etc.) y el año en que fueron tomados los datos.

Para mostrar las características de los profesionales encuestados, desarrollamos un cuadro que organiza y nos ubica en el relevamiento realizado, discriminando la cantidad de encuestas, su distribución por lugar, porcentajes relativos, rangos etarios, sexo, edad y estado civil de los participantes.

Cuadro 2: Encuestas. Desglose de información por Grupos

\begin{tabular}{|c|c|c|c|c|c|c|c|c|c|c|c|c|c|c|c|c|c|c|}
\hline \multirow[t]{2}{*}{ Grupo } & \multirow{2}{*}{ 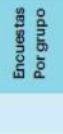 } & \multirow{2}{*}{ 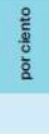 } & \multirow[t]{2}{*}{ H } & \multirow{2}{*}{ 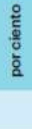 } & \multicolumn{4}{|c|}{ Edad } & \multicolumn{2}{|c|}{ 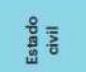 } & \multirow[t]{2}{*}{ M } & \multirow{2}{*}{$\begin{array}{l}\frac{8}{0} \\
\frac{5}{0} \\
\frac{0}{\circ}\end{array}$} & \multicolumn{4}{|c|}{ Edad } & \multicolumn{2}{|c|}{ 옳 $\overline{\frac{5}{5}}$} \\
\hline & & & & & $24 / 35$ & $36 / 45$ & $46 / 55$ & más 55 & c & s & & & $24 / 35$ & $36 / 45$ & $46 / 55$ & más 55 & c & s \\
\hline $\begin{array}{l}\text { Carmen de } \\
\text { Patagones } 2012\end{array}$ & 35 & 20 & 16 & 46 & 5 & 6 & 3 & 2 & 12 & 4 & 19 & 54 & 5 & 14 & 0 & 0 & 10 & 9 \\
\hline Concordia 2012 & 14 & 10 & 10 & 71 & 2 & 1 & 2 & 5 & 9 & 1 & 4 & 29 & 1 & 3 & 0 & 0 & 2 & 2 \\
\hline La Plata 2011 & 21 & 14 & 15 & $\pi$ & 6 & 9 & 0 & 0 & 10 & 5 & 6 & 29 & 2 & 2 & 2 & 0 & 4 & 2 \\
\hline Balcarce 2012 & 15 & 11 & 8 & 53 & 3 & 3 & 0 & 2 & 5 & 2 & 7 & 47 & 5 & 0 & 2 & 0 & 3 & 4 \\
\hline La Plata 2014 & 22 & 15 & 10 & 45 & 5 & 3 & 2 & 0 & 8 & 2 & 12 & 55 & $n$ & 0 & 1 & 0 & 3 & 9 \\
\hline Balcarce 2015 & 9 & 6 & 4 & 44 & 1 & 2 & 0 & 1 & 2 & 2 & 5 & 56 & 4 & 1 & 0 & 0 & 1 & 4 \\
\hline Caravelas 2014 & 13 & 9 & 10 & 77 & 3 & 5 & 2 & 0 & 8 & 2 & 3 & 23 & 3 & 0 & 0 & 0 & 3 & 0 \\
\hline Junin 2014 & 12 & 8 & 9 & 75 & 7 & 1 & 0 & 1 & 6 & 3 & 3 & 25 & 2 & 0 & 0 & 1 & 2 & 1 \\
\hline Rosario 2014 & 11 & 7 & 10 & 91 & 4 & 1 & 5 & 0 & 7 & 3 & 1 & 9 & 1 & 0 & 0 & 0 & 1 & 0 \\
\hline Total Encuestas & 152 & 100 & 92 & 61 & 36 & 31 & 14 & $n$ & 67 & 24 & 60 & 39 & 34 & 20 & 5 & 1 & 29 & 31 \\
\hline
\end{tabular}


1. Cuatro cohortes de la Maestría Procesos Locales de Innovación y Desarrollo Rural (PLIDER) donde se sondeó a alumnos que llegan desde todo el país, mayoritariamente extensionistas del INTA, para hacer su maestría en La Plata, Balcarce o Bahía Blanca. Para este trabajo fueron encuestados durante el curso Metodologías de intervención Social dos "ciudades"; en la sede La Plata fueron encuestadas las cohortes 2010 y 2014 y en Balcarce las cohortes 2012 y 2015. No se encuestaron cohortes en Bahía Blanca porque esa Sede no abrió durante el periodo estudiado.

42 2. En el caso del muestreo del Programa Pro Huerta, el instrumento fue utilizado en una capacitación que se realizó en Carmen de Patagones y nucleaba a los extensionistas del Programa que trabajaban para esa fecha en el Centro Regional Buenos Aires Sur (CERBAS) del Instituto Nacional de Tecnología Agropecuaria (INTA) y no eran alumnos de la Maestría PLIDER.

43 3. Respecto a los profesionales de Cooperativas encuestados, las tomas se realizaron durante tres capacitaciones a personas que en ese momento trabajaban en Cooperativas vinculadas a la Asociación de Cooperativas Argentinas (ACA), en áreas de influencia de las ciudades donde fueron encuestados. Tampoco eran alumnos de la Maestría PLIDER.

4. Por último se aplicó el instrumento a un grupo de extensionistas voluntarios sin Maestría en el marco de un Congreso de Extensión en Concordia.

Durante la encuesta los participantes tuvieron la oportunidad de contestar y analizar en forma individual sus respuestas respecto a los factores de riesgo psicosocial para luego pasar a compartirlo en un espacio común. Si bien la unidad de recolección y la de análisis fueron similares en un primer momento, los individuos, a medida que avanzó la investigación se sistematizaron las respuestas en forma colectiva por toma (Ciudad) y construimos una categorización por organizaciones.

Finalmente todos los cuestionarios son anónimos y tienen un pequeño cuadro inicial donde se incorpora la edad, estado civil y sexo de los profesionales, así como el lugar de toma de datos y año en que fueron realizadas. Esta información es importante para un posible análisis diacrónico de los resultados en trabajos posteriores.

\subsubsection{Análisis de correspondencias múltiples}

47 Cuando analizábamos en la planilla por colores los datos obtenidos por el cuestionario CoPsoQ-ISTAS 21 veíamos que similares colores en las respuestas incluían un rango de puntaje amplio en cada Apartado tanto para hombres o mujeres (por ejemplo en las celdas rojas el rango era 11 a 24 para Exigencias psicológicas, 20 a 0 para Trabajo Activo, 6 a 16 para Apoyo Social ó 23 a 0 para Inseguridad) y quisimos ver si esa amplitud de rango incluía comportamientos diferentes entre sí.

A partir de esa observación cargamos las respuestas por Apartado de los 152 cuestionarios y los sometimos a un análisis de correspondencias múltiples entre todos los grupos encuestados y los promedios de las respuestas de cada Apartado, estudiando las nubes de asociaciones entre individuos y modalidades, buscando cercanías de respuestas entre hombres y mujeres y entre grupos encuestados. En los Anexos presentamos las 24 salidas del Programa, graficando cuatro para cada apartado: la primera asocia variables por lugar y la segunda por lugar y modalidad de respuesta, esto se repite para varones y mujeres, por separado.

Laboreal, Volume $13 \mathrm{~N}^{\circ} 1$ | 2017 

número reducido de dimensiones, con la menor pérdida de información posible. En este aspecto su objetivo es similar al de los métodos factoriales, salvo que en el caso del análisis de correspondencias el método se aplica sobre variables categóricas u ordinales.

nos centramos en una tabla de contingencia de dos variables cualitativas, con una variable donde las categorías aparecen en filas y la otra representadas en columnas, el análisis de correspondencias consiste en resumir la información presente en las filas y columnas de manera que pueda proyectarse sobre un espacio reducido y representarse simultáneamente los puntos fila y los puntos columna, pudiéndose obtener conclusiones sobre las relaciones entre dos variables nominales $u$ ordinales de origen. En general se orienta a casos como éste, en los cuales una variable representa individuos y el resto son variables cualitativas que representan cualidades.

\subsubsection{Entrevistas semiestructuradas}

51

Otro instrumento de investigación que utilizamos fue la entrevista semiestructurada. La misma fue realizada a una muestra de 15 extensionistas del INTA, alumnos de la Maestría PLIDER, hombres y mujeres. Se los invitó a participar con la explicitación de que se garantizaría su confidencialidad y anonimato y su participación sería absolutamente voluntaria. Asimismo los nombres de los entrevistados han sido modificados para preservar su anonimato, no así su región de trabajo: Pampa Húmeda, Patagonia, Gran Buenos Aires, Noroeste Argentino (NOA) y Noreste (NEA).

Diseñamos un eje de ocho preguntas básicas relativas a como cambiaron los perfiles profesionales de los extensionistas en los últimos 20 años, si se sienten instrumentados para resolver las demandas socio productivas del territorio, como los afecta, si conocen que es un riesgo psicosocial y si las competencias desarrolladas durante la Maestría PLIDER ayudan a mejorar sus trabajos.

\subsubsection{Clínica de la actividad}

Denominamos Clínica de la Actividad al espacio de reflexión e interacción que se fue abriendo luego que los grupos encuestados respondieron el cuestionario CoPSoQISTAS21, pudiendo analizar los resultados obtenidos a nivel personal y grupal. La clínica de la actividad problematiza la organización del trabajo y provee herramientas para analizar las relaciones entre actividad y subjetividad (Clot, 1999), captando la importancia de la construcción de conocimiento por parte de los sujetos y grupos implicados.

54 Este ambiente de revisión de la actividad profesional se extendió durante una semana para los alumnos de la Maestría PLIDER, ya que se utilizó el soporte del curso Metodologías de Intervención Social. Para los restantes encuestados solo se dispuso de una tarde, en el marco de sus respectivas capacitaciones.

Laboreal, Volume $13 \mathrm{~N}^{\circ} 1$ | 2017 


\section{Los riesgos psicosociales según la encuesta y las entrevistas}

El modelo Demanda-control-apoyo social de Karasek y Theorell, (1990) -que utilizamos para comprender el comportamiento de los extensionistas- plantea que los efectos (positivos o negativos) de la actividad profesional son determinados por la combinación de dos dimensiones: las demandas psicológicas laborales y el control que el trabajador puede ejercer para elaborar estas demandas. Como respuesta a las exigencias que plantea el trabajo, se observa en los extensionistas un intento de control sobre las competencias como recurso para moderar las demandas.

Según Karasek, esta "latitud decisional" se traduce en grados de autonomía y habilidades como aprendizaje, creatividad y trabajo variado, siendo las oportunidades o recursos que la organización proporciona a estos profesionales para moderar o tomar decisiones sobre las demandas en la planificación y ejecución del trabajo. Sin embargo, el aumento de este tipo de autoridad decisional no siempre es sinónimo de un mayor control sobre las exigencias del trabajo y puede producir más stress, cuando los trabajadores no han recibido ni los medios, ni la formación necesarios (Vézina, et al., 2003).

57 La revisión realizada por Vézina sobre este modelo aplica a lo observado en nuestro trabajo de campo, donde puede verse que el modelo de Karasek no se comporta igual cuando los grados de latitud decisional en el territorio son mayores, sino amesetándose cuanto mayor es la complejidad de las demandas. En vez de generarse un aprendizaje activo y un mayor desarrollo de competencias, los extensionistas aumentan la exposición a los factores de riesgo psicosocial, se estresan y ponen en juego su salud. Cabe aclarar que en este modelo, “... el espacio de control en cuestión es, en verdad, predefinido" (Lacomblez \& Vézina, 2008, p.116)

En el siguiente cuadro visualizamos la exposición de los Extensionistas a los RPS, a través del diagnóstico que genera el Cuestionario CoPsoQ-ISTAS21. Los resultados estadísticos han sido traducidos a colores según el intervalo en el que cae la puntuación obtenida. Los números dentro de cada casillero son el promedio de la suma de resultados individuales obtenidos para cada ítem, por ejemplo para Exigencias Psicológicas, los 15 hombres encuestados de la cohorte PLIDER 2010 obtuvieron un promedio de 13 puntos, el cual entra en el rango del color rojo. 
Cuadro 3: Exposición a factores de riesgo psicosocial según cuestionario CoPsoQ-ISTAS21

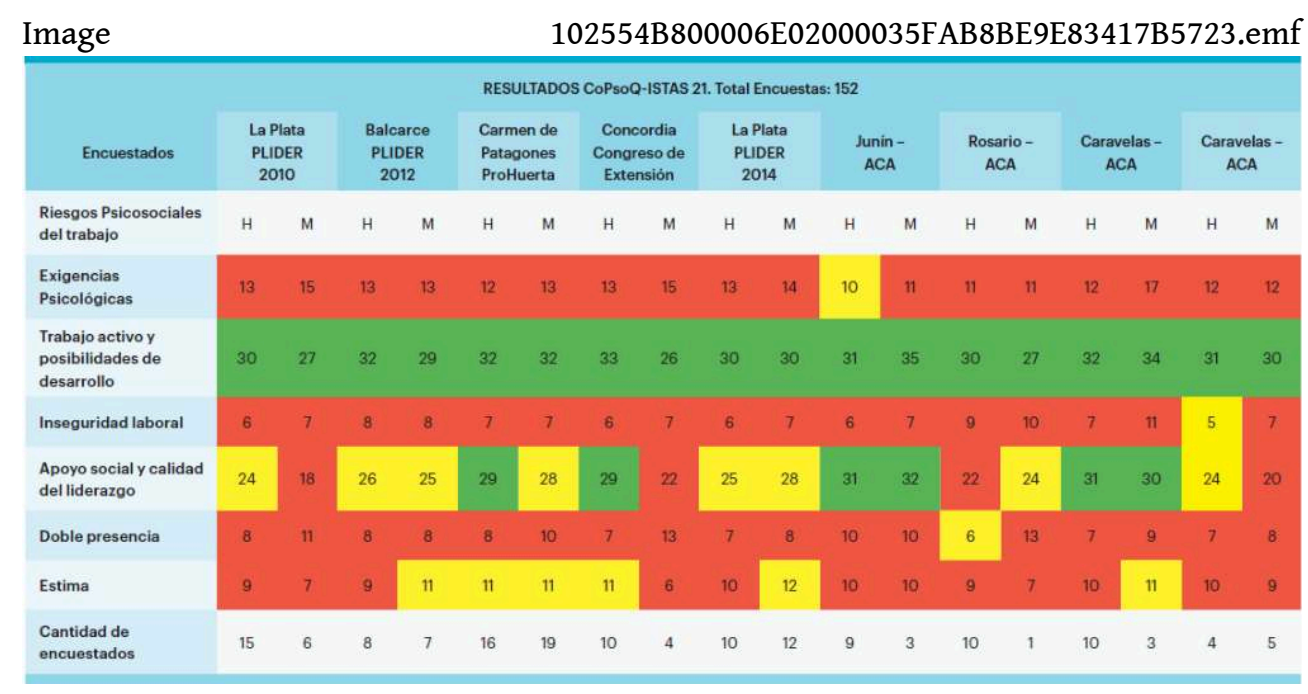

Una mirada general sobre el cuadro de resultados ISTAS nos llama la atención por mostrar mucha superficie roja y una línea verde de punta a punta.

El primer Apartado, Exigencias Psicológicas, se presenta casi todo en rojo, salvo para los Hombres de Junín ACA. El segundo Apartado, Trabajo Activo y posibilidades de Desarrollo, significativamente verde para los nueve grupos encuestados.

El tercer Apartado, Inseguridad laboral, se presenta casi todo rojo menos para el grupo de Hombres de la PLIDER 2015. El cuarto, Apartado Apoyo social y calidad del liderazgo, es donde más segregan las respuestas por la variedad de colores observados, correspondiendo los rojos y amarillos mayoritariamente a los extensionistas de INTA y los verdes a los profesionales de ACA.

El quinto Apartado -la Doble presencia- se observa todo rojo, menos para los Hombres de ACA Rosario y finalmente el sexto Apartado- Estima- se ve rojo con algunos amarillos mayoritariamente para las mujeres de ambas organizaciones.

3 A continuación, y a modo de síntesis, vamos a combinar los resultados de la encuesta ISTAS y las correspondencias múltiples con los emergentes más relevantes de las entrevistas que, a nuestro criterio, se relacionan íntimamente con cada uno de los Apartados.

En el Apartado 1 -Exigencias psicológicas- que consideramos el más importante por su influencia en el rol profesional, es interesante ver como aun teniendo una valoración de riesgo psicosocial alta para una abrumadora mayoría de los encuestados ISTAS - casi todos están en rojo en el cuestionario-las correspondencias múltiples nos muestran un gradiente donde los varones más exigidos son los de la Cohorte Balcarce 2012 (INTA) y los menos exigidos los varones de Junín ACA (únicos en amarillo para ese Apartado). Al contrario de los varones, las mujeres más exigidas son las de Caravelas (ACA) quizás por la menor formación frente a la tarea ya que algunas de las encuestadas no eran profesionales. Otra observación relevante respecto al desgaste emocional y a esconder las emociones, sondeado a través de las correspondencias múltiples, es que las puntuaciones más altas se observan en los Hombres de INTA, esbozando una relación con la pertenencia a tipologías organizacionales diferentes que desarrollaremos más adelante. 

paralelos, no sé cómo explicarlo específicamente, pero a veces me confunden un poco los objetivos que tiene la institución respecto al territorio, que a un agente de terreno le falten vehículos para atender a la gente, que es lo prioritario, mientras en el ámbito administrativo de la misma institución hay vehículos cero kilómetros parados... me parece una asimetría... Cuando por ejemplo la institución dice que lo prioritario es el pequeño productor, el desarrollo local, el desarrollo territorial..., pero de pronto se compran vehículos y ninguno está destinado al territorio... (Hombre, Patagonia) zonas de desastre, o lo complejo que resulta acompañar a productores en proceso de descapitalización, donde la demanda supera la formación de los profesionales y excede las competencias "explicitadas" para desarrollar su trabajo. Así, muchos entrevistados perciben en su trabajo una exigencia emocional similar a la de los cuidadores de ancianos o discapacitados.

71 “(...) en la zona hubo una inundación y pensás en el otro ya no tanto en lo productivo, sino en que quedó aislado, hay que llevarle abrigo, poner una camioneta a su disposición y ya no estamos pensando en una huerta, sino que estamos pensando en una asistencia" (Hombre, NEA (Noreste Argentino)).

72 La presencia de una situación conflictiva constituye un estresor importante teniendo como efecto inmediato un descenso en el logro de los objetivos de la organización y una disminución en la satisfacción del trabajador:

73 "Estoy muy preocupado porque las cosas me salgan bien, porque es mi trabajo, duermo un poco intranquilo a veces... Mi miedo es fallarle a la gente, estoy pensando más en el 
territorio, en la gente. Si yo planteo estas disconformidades... todos me dicen sí, tenés razón, pero no llegan las soluciones, cuando yo organizo algo y se me queda la camioneta, no puedo hacer nada, tengo que poner la cara y el problema es fallarle a la gente" (Hombre, NOA, (Noroeste Argentino)).

“(...) Creo que se nos exige mucho más de lo que profesional y humanamente podemos hacer.... en el caso de las áreas sociales, además de muchos conocimientos adicionales, se requiere un compromiso emocional muy alto, que en general el extensionista posee, pero que también lo lleva a descuidar actividades netamente referidas a la profesión y que después causan el reclamo de los productores o del propio organismo" (Mujer, NEA).

75 “(...) Sentimos bronca, muchísima. Sentimos bronca y desilusión no sólo institucional sino de las comunidades, del trabajo en terreno, de lo frágil que es todo; la institución siempre valoriza cuando le conviene o desvaloriza o minimiza cuando no le conviene y eso es duro. En este caso priorizaron una acción política y provincial a un trabajo de construcción de años, de fortalecimiento y con resultados concretos, nosotros teníamos resultados concretos, de acciones concretas y ejecutadas al cien por ciento. Entonces realmente sentís una fractura mental y sentimental también porque es imposible no hacer vínculos sentimentales, emocionales" (Hombre, NOA).

76 La Carga mental es otro factor de riesgo detectado. Se percibe de manera individual y esto hace que se encuentren diferencias interpersonales en relación a las formas en que se expresa y la intensidad en que se siente, en función de factores situacionales y características personales. (Arquer (de), \& Nogareda, 2004)

“(...) Siento un enorme cansancio al llegar a casa. Sin ganas de escuchar a la familia.... a veces tengo problemas para conciliar el sueño. Ansiedad. Contractura. Alergia" (Mujer, NEA).

78 Los profesionales con sensación de carga mental prolongada presentaron como consecuencia, fatiga mental, entendida como la disminución de la capacidad física y mental después de haber realizado un trabajo durante un período de tiempo determinado.

79 “(...) Sí, fumaba mucho más. El cansancio mental era mucho mayor también. Un cansancio brutal, pero que no impedía que siguiéramos movilizándolos" (Hombre, NOA).

80 En el Apartado 2 - Trabajo activo y posibilidades de desarrollo - significativamente verde para los nueve grupos encuestados, presenta un comportamiento diferencial para hombres y mujeres en las correspondencias múltiples. Mientras mayormente los hombres que trabajan en INTA ven su Trabajo como Activo y con posibilidades de desarrollo, son las mujeres de ACA las que tienen esta percepción, lo cual nos lleva a interpretar que ambas instituciones representan distintas tipologías y otorgan rangos de promoción y desarrollo profesional diferentes para hombres y mujeres, siendo más competitivo el INTA, lo cual favorece a los varones profesionales, y más integrativas las Cooperativas asociadas a ACA, lo cual beneficia a sus empleadas.

81 “(...) la decisión política de trabajar desde dimensiones sociales, territoriales y no sólo desde una lógica transferencista nos llevó a los extensionistas a desarrollar nuevas capacidades a fin de interpretar y dar respuesta a los territorios donde trabajamos" (Hombre, Patagonia).

82 "Me encanta ser agente de terreno..." (Mujer, Patagonia). 
83 "Creo que la posibilidad que me dio el INTA de hacer una Maestría me ayudó a comprender en el territorio muchos fenómenos que no comprendía. o tal vez los comprendía en forma tácita y el hecho de teorizar y conceptualizar los procesos me ayudó a poder describirlos en un trabajo. Dicen que el trabajo que hice me abrió la cabeza, me hizo ver de otra manera lo mismo (Hombre, Gran Buenos Aires).

El Apartado 3 - Inseguridad laboral - que se presenta casi todo rojo menos para el grupo de Hombres de la PLIDER 2015 en el ISTAS, nos muestra a través de las correspondencias múltiples que todavía y en general, los hombres temen más perder su trabajo y las mujeres temen más que les cambien las tareas en contra de su voluntad. También influye el enfoque diacrónico de la investigación en estos resultados, dado que las últimas cohortes PLIDER encuestadas se encuentran en una situación de estabilidad laboral dado el proceso que inició el INTA con la incorporación a planta de la mayoría de sus contratados, situación que no se veía en los comienzos de la toma de datos. "Sí, a nivel laboral constantemente es una inseguridad porque pienso en la relación costo beneficio... y hay momentos en que veo que hay más costos personales, porque además vivo sola, dejé mi familia, estoy resignando más cosas que en las que me beneficio o crezco personalmente, hay un crecimiento personal por supuesto... la vida no es fácil para nadie, pero siento inestabilidad al no saber hasta cuando me sostengo sola... mi futuro es una incertidumbre..." (Mujer, Patagonia).

"Yo siento que estoy instrumentado si me comparo con compañeros que vienen de otras ciencias. ... pero la complejidad sigue abrumándome, no es que estamos en una autosuficiencia que me deja tranquilo porque todo es muy cambiante. La situación de duda viene cuando a lo mejor me siento instrumentado, pero no estoy del todo seguro de estarlo... la institución... a veces no sabes bien si es para este lado que tenés que ir o hacia otro, silo que vos crees que hay que hacer está bien, si la instrumentación que uno tiene es la correcta... (Hombre, Pampa Húmeda).

“(...) de hecho yo estoy cursando una Maestría porque no sabía que era el territorio, no entiendo bien la idea, de que se trataba eso de lo que se hablaba todo el tiempo..." (Hombre, Gran Buenos Aires)

88 El Apartado 4 - Apoyo social y calidad del liderazgo - es donde más segregan las respuestas por la variedad de colores observados, correspondiendo los rojos y amarillos mayoritariamente a los extensionistas de INTA y los verdes a los profesionales de ACA. También nos muestra en ISTAS un comportamiento diferenciado entre hombres y mujeres, donde los primeros tendrían más capacidad de "lobby" o "actitud corporativa" dentro de cada Institución. En general las mujeres se sienten "un poco peor" por los desfasajes aún pendientes respecto a la igualdad de género, que se agudizan en ambientes masculinos y rurales, y quizás porque aún tenemos un comportamiento institucional menos corporativo, si bien las mujeres de la cohorte Balcarce 2012 se destacaron durante los cursos de la Maestría por su nivel de formación, competitividad y capacidad de liderazgo, lo cual queda expresado en este item.

89 Este Apartado también pone en evidencia las diferentes tipologías organizacionales analizadas, donde pareciera que tanto varones como mujeres de Cooperativas asociadas a ACA y el Programa Pro Huerta dentro del INTA (Patagones) tienen más claridad de rol en sus puestos de trabajo, mientras que los maestrandos PLIDER (INTA) están más atravesados por la verticalidad de las relaciones de poder, en entornos más 
competitivos que reducen el apoyo social de los pares y en los que es baja la calidad del liderazgo.

90 Las diferencias en el apoyo social entre pares y la calidad del liderazgo de los jefes impactan en las dimensiones del conflicto y la ambigüedad de rol, los grados de autonomía percibida, la comunicación y los estilos del liderazgo, emergentes que desarrollamos a continuación. En las entrevistas vemos como la organización presiona al extensionista a través del cumplimiento de las exigencias de la posición laboral pero al mismo tiempo se evidencia una falta de claridad sobre el trabajo que está desempeñando, los objetivos de éste y el alcance de las responsabilidades, generando ambigüedad de rol y no pocas veces, violencia psicológica sobre los profesionales.

91 “Tengo la ventaja que la institución me paga esta maestría, si bien todavía falta un proceso de decantación de todo lo que uno ve, me siento con más recursos y más cómoda en el terreno, pero a nivel institucional no me siento cómoda, siento que es contradictorio esto que a nivel nacional te otorguen fondos para capacitarte con un enfoque territorial, pero a nivel regional te limiten a un perfil más transferencista" (Mujer, Patagonia).

92 “(...) hay una competencia que muchos tienen a nivel institucional que es el hecho de conseguir apadrinamientos, que yo no la desarrollé, siento que laburo sola" (Mujer, Patagonia).

93 "Sí, perdimos la inocencia institucional, realmente te dan la espalda y te dejan en pampa y la vía. Así que no sólo nos dieron la espalda, sino que nos obligaron a corrernos del territorio". (Hombre, NOA)

94 El conflicto y la ambigüedad de rol, es otro factor de riesgo psicosocial que hace referencia a la existencia de demandas conflictivas o contrapuestas, donde aparecen simultáneamente exigencias de ambos lados que impiden a la persona una toma de decisión clara sobre qué hacer; o cuando las demandas y exigencias de la tarea son entre sí incongruentes o incompatibles para realizar el trabajo.

“(...) Cuando interactuamos con gente más grande nos dicen que las estructuras en las que trabajaban y los lineamientos eran otros. Generalmente refieren a cuestiones más transferencistas, más a la cuestión técnica. A veces lo plantean, depende con quien hable, como algo que era mejor... más simple, menos fuego cruzado". (Hombre, Pampa Húmeda)

96 La autonomía es otro de los factores de riesgo que consideramos fundamental para la satisfacción laboral y el bienestar de los profesionales. Se presenta como la capacidad del profesional para tomar decisiones relacionadas con su trabajo y controlar sus propias actividades. La posibilidad de organizar el trabajo, regular su ritmo o determinar el orden en el que se han de realizar las tareas parecen elementos importantes a primera vista y en un determinado grado de intensidad.

97 "Hay una tensión clara y surge en este empuje de la institución por trabajar con cuestiones del "desarrollo, pero éstos procesos demandan una energía muy grande y un tiempo que supera los tiempos políticos de la institución, entonces pasamos a ser muchas veces una amalgama entre fuerzas y tensiones, nos interpretamos como puentes y sin embargo muchas veces suele ser muy difícil que los procesos continúen o surjan... como si todo dependiera de nosotros" (Hombre, Patagonia).

98 “(...) La verdad que la sensación es de insatisfacción, algo de nerviosismo y en instancias muy complicadas contractura en el cuello. Aunque sigo sin fumar, sin beber, sin comer 
en exceso, supongo que en esas instancias uno puede llegar a dominarse, pero la taquicardia y nervios no" (Mujer, Pampa Húmeda).

“(...) Una sensación de insatisfacción porque obviamente es imposible lograr una solución a todo y llegar a tiempo a todas las demandas..." (Mujer, NEA).

A veces la obligación de hacer algo entra en conflicto con el poder actuar, hasta impedir actuar (Sznelwar, 2003). Cuando eso sucede la actividad no puede encontrar una salida y erupciona en trastornos psicosociales: absentismo, tensiones con los clientes, conflictos en los equipos, defectos, averías, dilación de tiempos, así como problemas de salud: stress, sufrimiento, acoso y depresión.

“(...) a veces me da cosa que se ilusionen, las caritas sonrientes... y uno piensa: ¿En que quedará todo esto? ..." (Mujer, NOA).

También observamos que algunos profesionales al verse impedidos de resolver los problemas de sus asistidos sólo consiguen salir de tales situaciones con el uso deliberado de distorsiones, encontrándose en situaciones de "no-go", lo que les genera un conflicto ético cuyos efectos nocivos son muy difíciles de contener y posiblemente generen riesgos para su salud.

Los problemas de comunicación observados, como las distorsiones, ambigüedades e incongruencias de los relatos, suelen ser un "caldo de cultivo" para conflictos interpersonales ya que muchas veces estas cuestiones emocionales son bloqueadas en la organización laboral, encontrando un canal en los vínculos que se establecen entre pares y con otros actores del territorio.

"Un colega me decía: fíjate cómo son las cosas, a vos te dio con un caño (su jefe) para que no te vayas a hacer una Maestría en desarrollo y ahora está hablando de desarrollo como una línea de acción muy fuerte para la Institución”. (Hombre, NEA)

5 También consideramos que los estilos de coordinación están en la base de numerosos problemas de relación en el trabajo. Algunas entrevistas dan cuenta de jefes con estilos autoritarios, provocando síntomas de stress, ansiedad y hostilidad. Del mismo modo otros dan cuenta de jefes con un estilo evitativo que dejan solo al profesional para tomar decisiones y sin recursos en el territorio.

“(...) desde que entré no tuve directivas; a nivel nacional tuve un curso de inducción en el que me explicaron cómo funcionaba el INTA. Pero a nivel local, en mi agencia, ninguna directiva, solo se apuntaba a trabajar más con las organizaciones y los pequeños productores. A los medianos y grandes se les aportaba información. El trabajo se limitaba al contacto con las organizaciones" (Mujer, NEA).

7 "Uno escribe tres proyectos diferentes para obtener fondos para poder trabajar y lo hace desde una mirada... pero no tienen buena recepción del coordinador de extensión, siempre con la sensación de que uno está mal orientado. Pero tampoco nadie te orienta, sí, es mucha tensión..." (Mujer, NEA).

8 Por el contrario, otros dan cuenta de jefes carismáticos que proponen metodologías de trabajo más participativas y permiten una mayor autonomía a sus colaboradores generándoles mayor bienestar y satisfacción laboral. A veces estos liderazgos desentonan en una cultura organizacional más verticalista.

9 El Apartado 5 -Doble presencia-muestra el reparto de las tareas del hogar entre los profesionales y sus parejas, donde es probable que influya el enfoque diacrónico de la investigación, mostrando cierta evolución en los últimos 5 años. Este ítem se observa 
todo rojo en el ISTAS, menos para los Hombres de ACA Rosario. Aquí juega mucho la edad de los encuestados, donde tanto los hombres como las mujeres mayores polarizan con sus parejas la responsabilidad de las tareas del hogar, siendo su distribución más equitativa entre los profesionales jóvenes.

Para el Apartado 6 -Estima- se ve rojo con algunos amarillos mayoritariamente para las mujeres de ambas organizaciones. Aquí vemos que las respuestas no están tan atravesadas por las tipologías organizacionales sino básicamente por la modalidad del liderazgo de los jefes inmediatos. Con las correspondencias múltiples observamos que los hombres son los que más sufren la falta de reconocimiento, la falta de apoyo en situaciones difíciles o el trato injusto, quizás por liderar más procesos institucionales y quedar más expuestos, por ser menos demostrativos o propensos a reclamar, y por tener mayoritariamente la responsabilidad de mantener el empleo. Aunque sus colegas mujeres adoptan una posición más subordinada y de menores responsabilidades, se destaca el comportamiento del grupo de 7 mujeres de la cohorte PLIDER 2012 Balcarce, que tuvieron un posicionamiento de género muy fuerte, si bien no quedo expresado en las entrevistas fue evidenciado durante todo el proceso. También es significativo el amarillo tanto para Hombres como Mujeres del grupo Carmen de Patagones, donde su mayor instrumentación en dinámicas grupales, así como la pertenencia institucional, pueden reforzar su Estima en los grupos de pertenencia.

111 “(...) Yo trabajé en un plan de desarrollo estratégico, infinidad de reuniones con actores locales y cuando paraba pensaba que estaba trabajando en el Ministerio de Desarrollo Social no en INTA. Mi jefe alentaba y acompañaba el proceso, pero uno no puede dejar de mirar" (Hombre 4, Pampa Húmeda).

112 "Las competencias hacia lo institucional tienen que ver casi con una cuestión de estar bien yo, porque la desvalorización tiende a bajarte... si no estás bien vos... Fue duro, ahora ya no me siento mal, no lo tengo que ejercitar tanto, me siento. Dicho en lenguaje simple, le doy cada vez menos "pelota" ... De hecho, pasa un tiempo largo hasta que entienden que no soy ingeniero y eso a veces me hace pensar qué imagen estoy dando" (Hombre, Pampa Húmeda).

113 "Después de estar en esa situación tan rústica (viajo $120 \mathrm{~km}$ para ir a una reunión, caminos con hielo y nieve...), me pude adaptar a una cultura totalmente diferente a la mía. Como que ya hice la base en las condiciones más desafiantes y espero que eso me legitime en el momento de coordinar proyectos" (Mujer, Patagonia).

114 “La (maestría) PLIDER me ayudó a entender que las visiones son infinitas y que las exigencias son igualmente sentidas entre compañeros y que en general hablamos poco de eso. Me dio un margen de compresión para entender emocionalmente muchas acciones que me han ayudado a tener mejor llegada tanto a nivel del territorio como en la propia institución y me ha enseñado a esperar los tiempos del desarrollo, bajar el nivel de ansiedad que creo es una de las causas de las frustraciones cotidianas: juno quiere ver los resultados!" (Mujer, NEA).

115 "No sé si puedo decir que el taller disminuye el riesgo psicosocial, pero me siento mucho mejor conmigo y con mi trabajo" (Mujer, NOA).

116 Por último, la mayoría de los encuestados siente que, si bien hay un reconocimiento profesional externo, en el territorio, la organización laboral no estimula la estima de los profesionales. Por el contrario, se observan dificultades para reconocer y evaluar el carácter procesual de sus trabajos, lo cual los invisibiliza. 
117 “Ciertamente, el reconocimiento profesional muchas veces no está en relación al esfuerzo realizado" (Mujer, NEA).

“(...) Estoy expuesta a la burocracia, a que dejen que el extensionista ponga la cara sin acompañarlo, al no reconocimiento de las labores que se van desarrollando, a cortar o no apoyar los procesos por falta de presupuesto, a las altas demandas territoriales en distintas temáticas productivas, a la sectorización política que implica trabajar en instituciones estatales" (Mujer, NOA).

\section{Discusión de los resultados}

Contestando la pregunta inicial sobre qué les pasaba a los extensionistas que se los notaba tan estresados, en función de los datos obtenidos y los emergentes sistematizados, llegamos a las siguientes conclusiones:

El trabajo como promotor de desarrollo profesional y social

Para los extensionistas la promoción laboral y social, la expectativa de crecer profesionalmente y el hecho de desempeñar un trabajo acorde con su formación y preparación, constituyen un incentivo, que los compensa, y aumenta su importancia conforme mayor es su reconocimiento social, visibilidad y cualificación profesional.

\section{La tensión en la posición de enlace}

La mayoría de los profesionales comunica stress laboral por las elevadas Exigencias Psicológicas que sienten al sostener un trabajo que tiene sentido para ellos, donde se manejan con un buen margen de autonomía, aplicando competencias previas y desarrollando nuevas, en el que sienten que se desarrollan pero que les implica un gran esfuerzo emocional.

Los extensionistas, última frontera de la organización, ocupan una "posición de enlace" entre la vida institucional y los territorios donde intervienen, convirtiéndose en un fusible, en un punto vulnerable que colapsa ante el aumento de tensión o intensidad, para proteger al sistema.

\section{El solapamiento de las demandas y el conflicto por ambigüedad de rol}

Estos perfiles profesionales se instalan en una "zona de clivaje", donde el cambio de condiciones los expone a sostener diversas tensiones. Hacia el territorio, el extensionista desarrolla un vínculo intersubjetivo, más íntimo y silencioso con el productor-asesorado, quien establece con el profesional una demanda emocional, propia de alguien "a cuidar" en el sentido de reclamar atención, información, tiempo, soluciones, presencia, contención emocional, subsidios, contactos, créditos, capacitaciones, acceso a redes, etc. y volverlo coparticipe de los resultados que se obtienen.

Cuando la posición organizacional del extensionista es más territorial y cuanto mayor el compromiso de su subjetividad con la acción colectiva, mayores serán los desajustes de este vínculo dilemático entre la organización y el territorio, que haciendo crisis en la persona del extensionista, aumenta su exposición a los factores de riesgo psicosocial.

Institucionalmente, los extensionistas deben actuar con lógica política, aquella imperante en la institución laboral, y bajo relaciones de poder verticales. Acreditar previsibilidad e informar burocráticamente sobre todos sus movimientos. A la vez y para lograr cierta capacidad de innovación, estos profesionales deberán sortear las 
trampas del esquema burocrático y articular con estructuras organizativas mucho más flexibles, desarrollar otros conocimientos y destrezas, y articular las diferentes lógicas que separan la organización del territorio. 
variabilidad. Esta regulación incide en la autonomía percibida, que cuando se intenta resolver cuestiones complejas en soledad, toma una intensidad que agudiza las consecuencias del vínculo entre la autonomía y el stress. Así, una Intensidad excesiva del contexto de trabajo, sumada al natural y paulatino envejecimiento personal, podría hacer necesaria una mayor regulación para la adaptación, generando un mayor desgaste personal por la intensidad de la actividad. Esta observación es congruente con la necesidad de "rejuvenecer" la planta profesional en ambas organizaciones, ya sea por mayor edad en el caso de INTA o por alta rotación en ACA, ya que en la posición de enlace los extensionistas sufren mayor erosión en contacto con los actores del territorio.

La cultura organizacional de pertenencia y la generación del riesgo psicosocial

mayormente los extensionistas tienen un comportamiento similar en ambas organizaciones, se observa que los profesionales de INTA se mueven en un entorno más competitivo, recibiendo menos apoyo social de sus jefes y pares mientras los profesionales de las Cooperativas asociadas a ACA tienen una mejor calidad de liderazgo, mayor cohesión entre pares y cercanía con sus superiores. También encontramos diferencias entre orígenes institucionales respecto al reconocimiento que obtienen desde la comunidad, donde los profesionales de INTA tienen una mayor autovaloración, principalmente las mujeres, en relación a sus colegas de ACA, probablemente por el mayor reconocimiento social hacia la institución de pertenencia.

Coexisten en ambas organizaciones laborales crecientes grados de burocratización, en INTA se observa cierta centralización de recursos, la formalización de los procesos decisorios, la dilución de la responsabilidad a través de las cadenas de mando y a la vez una excesiva personalización de la responsabilidad en el territorio. Con el tiempo, se genera una brecha profunda entre la estructura organizacional y la prestación de servicios al sector al que van dirigidos.

141 También ponemos en juego el factor "escala organizacional", donde el INTA es una organización empleadora de orden nacional, de imagen "jerárquica y poderosa" ante el profesional y la comunidad, que expresa con más crudeza la burocratización de su estructura, comparada con las Cooperativas locales asociadas a ACA, con estructuras autónomas de menor escala, donde estas características se muestran atenuadas.

\section{La división del trabajo y el verdadero cliente}

La organización y división del trabajo y los estilos de coordinación afectan la productividad, el nivel de satisfacción y la salud de estos profesionales. Muchos de los extensionistas relevados afirman realizar acciones erráticas en los territorios debido a la falta de soporte y retroalimentación, o por la inadecuada planificación de sus equipos de trabajo en el mediano y largo plazo. Estas acciones son parcialmente aprovechables por la falta de continuidad y solapamiento institucional en la implementación de las políticas. Aquí vemos cómo opera la división del trabajo: se actúa sin el actor competente, llevando a los profesionales a decidir sin tener en cuenta dimensiones que están fuera de sus competencias o a no intervenir sobre temas en los que no se consideran autorizados. Las metodologías grupales propuestas por los programas de intervención del INTA han dejado expuestos a muchos profesionales que, mayormente Ingenieros Agrónomos, carecen de las competencias necesarias para coordinar procesos grupales; y aquellos formados en profesiones con las habilidades sociales 
necesarias, adolecen de las competencias técnicas productivas para encauzar los proyectos grupales, ambos perfiles desbordados por la complejidad de las demandas.

Desde una lógica económica, el productor es un "cliente" que no le paga al extensionista, cuyo asesoramiento técnico productivo esta subsidiado por las agencias de extensión con diversas finalidades:

- acompañar la venta de insumos y fidelizar a sus clientes en el caso de las cooperativas, o

- captar, proteger y agrupar pequeños y medianos productores en el caso del INTA

Además, la capacidad de respuesta del profesional está sujeta, entre otras cosas, a las relaciones que establece con su organización laboral, quien le "extiende" saberes y recursos en función de la visualización del problema y su capacidad de negociación política. Así, la organización laboral funciona como el "verdadero prestatario" del extensionista ya que ahí se valida y retribuye su trabajo, lo que implica que muchas veces las tensiones se terminen resolviendo en función de su empleador, lo que puede vivenciarse como un conflicto personal.

\section{El impacto del Curso Estrategias de Intervención Social}

Para nosotros resultó una sorpresa que, tras responder el cuestionario CoPsoQ-ISTAS21 de Riesgos Psicosociales del trabajo, los alumnos de la Maestría PLIDER hubieran quedado movilizados y con necesidad de discutir el asunto en clase. Esta situación la presentamos como nueva información empírica, ya que, como consecuencia de este interés, el curso evolucionó en el tiempo respondiendo a las necesidades emergentes de los profesionales con una readecuación de su oferta formativa, mudando desde la posición final, última en la currícula, al primer lugar y ser la oferta curricular que abre la Maestría desde hace tres cohortes: La Plata 2014, Balcarce 2015 y Bahía Blanca 2016.

150 Esto implicó cambios paulatinos en los contenidos y métodos pedagógicos, producto de varios factores a detallar:

151 1. Para los extensionistas podemos hablar de la verificación, en ese espacio, de un "género profesional" como conjunto de obligaciones que, sin proponérselo, se impone el grupo profesional para poder alcanzar el objetivo, incluso en contra del modus operandi prescrito. (Fernández, \& Clot, 2007). Además, es un conjunto de evaluaciones de las situaciones compartidas por los profesionales, que sirve para organizar de forma implícita su actividad, permitiendo la irrupción de una clínica de la actividad (Clot, 1999) donde pueden trabajar las tensiones propias de la posición de enlace, sus consecuencias no deseadas y los efectos colaterales del trabajo emocional que requieren atención.

152 2. Para el Curso, convertirse en una oferta dinámica que abre la posibilidad de transformarse en función de las demandas en terreno de los profesionales.

153 3. Para el Programa de la Maestría PLIDER, la posibilidad de incluir los riesgos psicosociales como tema de interés manifestado por los propios interesados, y reconocido por los directivos, como se refleja en los cambios de contenidos y posición de la materia en la formación.

154 A través de la experiencia sistematizada mostramos este proceso como un resultado práctico no esperado de la Maestría, que justifica la inclusión de una oferta explícita denominada Clínica de la Actividad en la actualización permanente de los 
profesionales, resolviendo un conflicto estructural entre el diseño académico de la propuesta original francesa y la realidad de los extensionistas argentinos.

Para concluir, la utilización de las entrevistas semiestructuradas individuales, una vez finalizados los cursos, representa una etapa en la investigación que apuntó a la implicación progresiva de unos y otros, dando testimonio voluntario de sus propias reflexiones, las cuales reforzaron las conclusiones de nuestro trabajo. Lo que subyace en ellas, para lo cual los entrevistados "no tienen palabras", es la evidencia tácita del proceso por el cual el elevado grado de autonomía del que disponen para adaptarse a la exigencia laboral incrementa su exposición personal a niveles de riesgo.

\section{Comentarios finales}

¿Por qué se evalúan tan poco los riesgos psicosociales? En primera instancia por la dificultad de su evaluación, la falta de un mayor desarrollo de técnicas adaptadas a distintos contextos organizativos, cierta confusión conceptual de alguna de sus variables, su relativa novedad dentro del marco preventivo pero fundamentalmente por la clara preponderancia que se le otorga a los factores de seguridad músculoesqueléticos en detrimento del resto.

Esta investigación se nutrió de dos miradas complementarias: desde la ergonomía, el Riesgo psicosocial funciona como una advertencia saludable ya que remite a la cuestión de la actividad y nos permite enfocar las cuestiones organizacionales del contexto de trabajo y su íntima relación con las personas estudiadas, mientras que la clínica de la actividad nos ayudó a posicionarnos en el conflicto intersubjetivo del profesional con los vínculos establecidos.

Los resultados de nuestra investigación prueban que los extensionistas están fuertemente expuestos a los factores de riesgo laboral de origen psicosocial, y que la alta valoración que tienen de su trabajo es el gran convocante que justifica su exposición a ellos.

Los servicios de Extensión en Argentina buscan articular las exigencias de una relación basada en el servicio profesional, profundamente intersubjetiva, con una lógica de estandarización de audiencias a través de los Programas de Intervención. Cuando la forma de hacerlo no está suficientemente desarrollada, la resolución queda en manos del extensionista y las tensiones se transforman en el centro de su actividad. Los extensionistas, más allá de su compromiso y sentido del servicio, como profesionales "de frontera" han sido históricamente desvalorizados, entre otras formas, al no proveerles los medios apropiados para tal exigencia. El profesional vive este destrato con incertidumbre, no sabe qué se espera de él, y si la ambigüedad es sostenida en el tiempo se transforma en una amenaza para su salud.

Esperamos que este trabajo aporte al bienestar y aumento de la calidad de vida de los profesionales, además de ser un espacio formativo en tecnologías sociales y funcionar como un dispositivo para la revisión de la práctica profesional, ya que las exigencias que plantea la posición de "enlace" erosionan la actividad y deterioran potencialmente la salud de los extensionistas en el territorio.

161 También creemos que este trabajo es un aporte a la evaluación institucional, dada la invisibilidad de la realidad del trabajo y del compromiso de la subjetividad en los vínculos establecidos por los extensionistas, a los que les dio una voz para canalizar las 
demandas por el sufrimiento en el trabajo y a la vez un espacio para gestionar los recursos de la actividad.

Las organizaciones empleadoras disponen de un instrumento que pone en valor los procesos desplegados desde una evaluación más comprensiva que aporta a la prevención primaria de la salud al cuestionar las formas de organización del trabajo y el silenciamiento de su impacto sobre la salud de los profesionales, a la vez que propone instrumentos personales y organizacionales para reducir los PSR.

\section{BIBLIOGRAFÍA}

Arquer (de), M. I., \& Nogareda, C. (2004). Carga mental de trabajo: diseño de tareas (Notas técnicas de prevención, 659). Madrid: INSHT.

Baudelot, C., \& Gollac, M. (comp.) (2011). ¿Trabajar para ser feliz? La felicidad y el trabajo en Francia. Buenos Aires: Miño y Dávila/CEIL/Trabajo y Sociedad.

Bass, D. M., Tausig, M. B., \& Noelker, L. S. (1988-1989). Elder impairment, social support and caregiver strain: A framework for understanding support's effects, Journal of Applied Social Sciences, Vol. 13 (1), pp. 80-117.

Clot, Y. (1999). La fonction psychologique du travail. París: PUF.

CoPsoQ21. (2000). Cuestionario psicosocial de Copenhague revisado en Barcelona para habla hispana: ISTAS. Ver en www.istas.ccoo.es/descargas/m_metodo_istas21.pdf

Davezies, P. (2009). Dérèglements organisationnels, santé, pouvoir d'agir. Retirado en Julio, 22, 2010 de http://www.comprendre-agir.org/images/fichier dyn/doc/2009/

dereglements_organisationnels_davezies_2009_chsct.pdf

Fernández, G., \& Clot, Y. (2007). Entrevistas en auto-confrontación: un método en clínica de la actividad. Laboreal, 2, (1), 15-19. http://laboreal.up.pt/revista/artigo.php?

id=37t45nSU547112298742:372391

Gollac, M. \& Volkoff, S. (2000). Les conditions de travail. Paris: Éditions La Découverte.

Grandey, A. A. (2000). Emotional regulation in the workplace: A new way to conceptualize emotional labor, Journal of Occupational Health Psychology, 5 (1), pp. 95-110. https://doi.org/ 10.1037/1076-8998.5.1.95

Lacomblez, M., \& Vézina, N. (2008). Karasek. Laboreal, 4, (2), 114-117. http://laboreal.up.pt/ revista/artigo.php?id=48u56oTV6582234528993784382

Maggi, B. (2006). "Bienestar". Laboreal, 2, (1), 62-63. http://laboreal.up.pt/revista/artigo.php? id=37t45nSU5471122651:3322771

Mintzberg, H. (1989). Diseño de organizaciones eficientes. Buenos Aires: El Ateneo.

Moncada, S., Llorens, C., \& Kristensen, T.S. (2002). Manual de la versión media del método ISTAS.

http://www.istas.ccoo.es/descargas/m_metodo_istas21.pdf 
Moncada, S., Llorens, C., Navarro, C., \& Kristensen, T.S. (2005). ISTAS21: Versión en lengua castellana del Cuestionario Psicosocial de Copenhague (COPSOQ). Archivos de Prevención de Riesgos Laborales, vol. 8, nำ, pp.18-29.

National Research Centre for the Working Environment (NRCWE) (2000). Retirado en Marzo, 9 de 2010 de http://www.arbejdsmiljoforskning.dk/en/om-os

Schwartz, Y. (2005). Actividad, Laboreal, 1, (1), pp. 63-64 http://laboreal.up.pt/revista/artigo.php? $\underline{\mathrm{id}=37 \mathrm{t} 45 \mathrm{nSU} 5471123588698: 54}$

Sznelwar, L. I. (2003). Working and impediment: The Causes and Consequences of inadequate Task Content and Work Organisation. In XVth Triennal Congress of the International Ergonomics Association, 7th Joint Conference of Ergonomics Society of Korea/Japan Ergonomics Society.

Vézina, N., Stock, S.R., Simard, M., Saint-Jacques, Y., Boucher, M., Lemaire, J., \& Trudel, C. (2003). Problèmes musculo-squelettiques et organisation modulaire du travail dans une usine de fabrication de bottes, Phase 2: Étude de l'implantation des recommandations. Montréal: Collection Études et Recherches - IRSST, R-345. http://www.irsst.qc.ca/fr/_publicationirsst_100006.html

\section{RESÚMENES}

Evaluamos la exposición a factores de riesgo psicosocial en agentes de los Servicios de Extensión Agropecuaria en Argentina. Encuestamos 152 extensionistas de dos organizaciones y diversas provincias argentinas con el instrumento CoPsoQ-ISTAS 21 y debatimos los resultados con 67 de estos profesionales durante un curso de posgrado de la Maestría PLIDER. Este curso funciono para los extensionistas como una Clínica de la actividad, permitiéndoles gestionar sus propios recursos ante el stress sostenido provocado por la exposición a los factores de riesgo. La investigación se reforzó con 15 entrevistas en profundidad.

Los resultados muestran una fuerte exposición a los factores de riesgo psicosocial donde aquellas causas que prevalecen derivan de la forma de organización del trabajo en cada institución de pertenencia, el elevado componente emocional del rol laboral, y la diferencia entre hombres y mujeres en cuanto a la autonomía en su realización, las exigencias inherentes a la posición de enlace organizacional, el conflicto por la ambigüedad del rol y su carga mental.

Avaliámos a exposição a fatores psicossociais de risco na atividade profissional de técnicos do setor agropecuário dos Serviços de Extensão, na Argentina. Conduzimos a análise com 152 extensionistas de duas organizações, e várias províncias argentinas, com o instrumento CoPsoQistas 21 , e debatemos os resultados com 67 destes profissionais durante um curso de pósgraduação do mestrado PLIDER. Este curso funcionou para os extensionistas como uma Clínica de atividade, permitindo-lhes gerir os seus próprios recursos face ao stress causado pela exposição aos fatores de risco. A investigação foi reforçada com 15 entrevistas em profundidade.

Os resultados mostram uma forte exposição a fatores psicossociais de risco onde as causas predominantes derivam da forma de organização do trabalho em cada instituição de pertença, da elevada componente emocional associada ao papel desempenhado em contexto de trabalho, e à diferença entre homens e mulheres em termos de autonomia na sua realização, dos requisitos inerentes à posição hierárquica, do conflito associado à ambiguidade do papel desempenhado e, por consequência, à carga mental.

Nous avons évalué l'exposition à des facteurs de risque psychosociaux chez des agents des services de vulgarisation agricole en Argentine. L'analyse, concernant 152 agents appartenant à deux organisations opérant dans plusieurs provinces argentines, a recouru à l'instrument CoPsoQ-ISTAS 21. Les résultats ont été débattu avec 67 de ces professionnels durant un cours de 
la Maîtrise PLIDER. Le cours a ainsi fonctionné pour ces techniciens comme une Clinique de l'activité, leur permettant de gérer leurs propres ressources face au stress causé par l'exposition aux facteurs de risque repérés. La recherche a été enrichie par 15 entretiens en profondeur.

Les résultats montrent une forte exposition aux facteurs de risque psychosociaux, et les causes prédominantes découlent $\mathrm{du}$ mode d'organisation du travail dans chaque institution d'appartenance, de la forte composante émotionnelle du rôle professionnel, de la différence entre hommes et femmes en termes d'autonomie de réalisation, des exigences inhérentes à la position de la hiérarchie, du conflit quant à l'ambiguité du rôle assuré et de sa charge mentale.

We evaluated the exposure to psychosocial risk factors in agents of the Agricultural Extension Services in Argentina. We surveyed 152 extension workers from two organisations and several Argentine provinces with the CoPsoQ-ISTAS 21 instrument and discussed the results with 67 of these professionals during a postgraduate course of the PLIDER Masters. This course worked for the extension workers as a Clinic of the activity, allowing them to manage their own resources when facing stress caused by exposure to risk factors. The research was reinforced with 15 indepth interviews.

The results show a strong exposure to psychosocial risk factors, where the prevailing causes derive from the form how work is organised in each host institution, the high emotional component of the job role, and the difference between men and women in terms of autonomy in its performance, the inherent demands of the hierarchical position, the conflict of the ambiguity of the role and, consequently, its mental load.

\section{ÍNDICE}

Mots-clés: risques psychosociaux, agents de vulgarisation agricole, Argentine

Palabras claves: riesgos psicosociales, extensionistas agropecuarios, Argentina

Keywords: psychosocial risks, agricultural extension workers, Argentina

Palavras-chave: riscos psicossociais, técnicos agropecuários, Argentina

\section{AUTOR}

\section{ROSSANA CACIVIO}

Facultad de Ciencias Agrarias y Forestales.

Universidad Nacional de La Plata -Av. 60 y 119 s/n (1900) La Plata.

Provincia de Buenos Aires, Argentina.

rcacivio@agro.unlp.edu.ar 